\title{
Low-voltage light beam steering by planar dual- domain nematic liquid crystal deflector
}

\author{
I.I. Rushnova, O.S. Kabanova, E.A. Melnikova, A.L. Tolstik \\ Physics Department \\ Belarusian State University \\ Minsk, Belarus, \\ Rushnova@bsu.by
}

\begin{abstract}
The paper presents a low-voltage dual-domain liquid-crystal element with planar and homeotropic director orientations that performs the functions of an optical deflector. Its operation principle is based on the revealed phenomenon of the spatially displaced refractive interface in liquid crystal cells with a patterned current-conducting layer under the effect of a low-frequency electric field. Linear displacement of a light wave reflected from the rectilinear interface is demonstrated experimentally and comes to $\sim 7 \mu \mathrm{m} / \mathrm{V}$. Optical components of this type offer much promise for the development and manufacturing of highly functional integrated photonics devices and of advanced control systems for optical radiation.
\end{abstract}

Keywords—optical switches, liquid crystal devices, liquid crystal deflectors, light beam steering.

\section{INTRODUCTION}

Liquid crystals (LC) exhibit numerous unique properties with great potentialities both for the fundamental research and innovative applications of photonics. At the present time LCs are extensively studied and widely used in information recording, processing, storage, and display devices due to their high anisotropy of the refractive index controllable by means of the applied low voltages $[1,2]$. Of particular interest is the development of optical LC switches because they offer possibility for light beam steering without the intermediate signal transformation to the electronic form and for various applications in telecommunications, networking, computers, servers, instrumentation, etc. [3]. In the recent decade much attention has been given to planar nematic liquid crystal (NLC) commutators with a dual-domain orientation of the director as they feature high reliability, without the use of additional optical elements, and simplicity of manufacturing procedures $[4,5]$. Their operation principle is based on total internal reflection (TIR) of a light wave from the interface between LC domains with orthogonal orientations of the director. It should be noted that in the majority of publications devoted to planar LC switches the authors consider mainly the features of spatial separation of the orthogonally polarized light beams at the refractive interface. Up to now, the possibility to realize light beam steering in planar LC cells has not been considered.

The present work is devoted to the design, manufacturing, and investigation of dual-domain LC elements with the electrically-switchable refractive interface which realize steering of linearly polarized light beams.

\section{LC CELls: Design AND FABRiCATION PROCESS}

The developed optical LC deflector represents a planaroriented LC cell of the sandwich type, with a rectangular patterned conductive layer of chromium on one of the substrates (in particular cases the surface filling factor was $1 / 2$ ), to provide the formation of two LC domains with orthogonal orientations of the director $\mathbf{n}$ due to the effect of an external electric field.

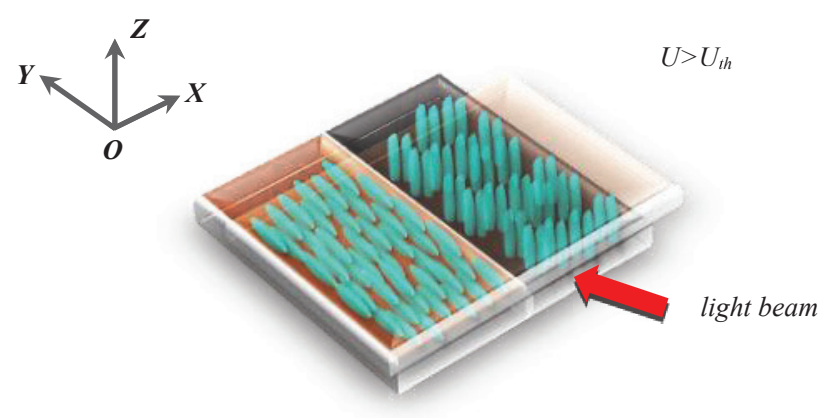

Fig. 1. Diagram of LC cell with a patterned rectangular conductive layer.

The refractive interface in a nematic LC layer is formed due to the creation of planar and homeotropic orientations of the director in a dual-domain element. Switching of the electrically-induced refractive interface is realized by application of the low-frequency voltage to the planar-oriented LC cell with a patterned electrode. At the voltage-on-state in the region of the patterned electrode the molecules of LC move in the $O Z$ direction, leading to the formation of a $\mathrm{LC}$ domain with the homeotropic director orientation. In the adjacent region of the cell, due to the absence of a conductive layer, there is no potential difference and, as a result, a planar orientation of LC in the domain under study is retained. In this way the formed LC domains with mutually orthogonal orientations of the director are characterized by different refractive indices for linearly-polarized radiation.

The initial planar molecular orientation of a nematic LC in cells was created by the contactless photoalignment method [6]. The azo dye AtA-2 synthesized at the New Materials Chemistry Institute of the NAS of Belarus was selected as a photoaligned material [7]. The retained electrode of chromium occupying a part of the substrate surface was formed by the method of photolithography. 
The quality of the initial molecular orientation in LC was improved using the original technology based on the specially developed composite structure comprising the buffer layer of nylon-6 and the photoaligned azo dye AtA-2 [8]. In the process of work we have used the nematic liquid crystal with the refractive index anisotropy $\Delta n=0.156$ at the wavelength $\lambda=632.8 \mathrm{~nm}\left(n_{e}=1.687, n_{o}=1.531\right)$. The LC layer thickness in the manufactured elements was $20 \mu \mathrm{m}$, offering the possibility to control the refractive interface by low voltages. The threshold voltage of the LC director reorientation was $U_{t h}=1.0 \mathrm{~V}$. The quality factor of LC orientation $q$ in the manufactured LC cells with a sublayer of nylon-6 was ranging $q=0.996-0.998$ at the wavelength $\lambda=632.8 \mathrm{~nm}\left(q=\left(T_{\max }{ }^{-}\right.\right.$ $\left.T_{\min }\right) /\left(T_{\max }+T_{\min }\right)$, where $T_{\max }-$ transmission factor of the LC sample in parallel polarizers $T_{\min }$ - transmission factor of the LC sample in crossed polarizers).

\section{EXPERIMENTAL RESULTS AND DISCUSSION}

When the LC element is subjected to the effect of an external electric field $\left(U>U_{t h}\right)$ and the wave is propagating with the polarization vector $\mathbf{E} \| O Z$ in the region of incidence (at the side of the opaque electrode and of the homeotropic director orientation), an extraordinary wave excited within the LC layer has the refractive index $n_{e}$ as directions of the vectors $\mathbf{E}$ and $\mathbf{n}$ are coincident. In the adjacent region, where there is no opaque electrode and no molecular reorientation, an ordinary wave with the refractive index $n_{o}$ is excited. In this way a linearly polarized light wave with the polarization vector $\mathbf{E} \| O Z$, on its propagation from the region with a higher refractive index to that with a lower one may be subjected to total internal reflection. To this end, the incidence angle on the interface should be higher than the critical angle:

$$
\alpha_{\mathrm{cr}}=\arcsin \left(n_{o} / n_{e}\right)
$$

The critical incidence angle $\alpha_{\text {cr }}$ for the interface of the used NLC was $\alpha_{\mathrm{cr}}=64.9^{\circ}$. The wave having the polarization vector $\mathbf{E} \| O X$ in the region of the light beam incidence is characterized by the ordinary refractive index $n_{o}$, whereas in the adjacent region - by the effective refractive index that is given by the following formula:

$$
n_{e f}=n_{o} n_{e} /\left(n_{o}^{2} \cos ^{2} \alpha+n_{e}{ }^{2} \sin ^{2} \alpha\right)^{1 / 2}
$$

On propagation from the region with a lower refractive index $n_{o}$ to the region with a higher refractive index $n_{e f}$, at the interface the light wave $(\mathbf{E} \| O X)$ is divided into the reflected and the refracted waves, the reflection factor being no greater than $0.25 \%$.

Being focused by the $10 \mathrm{X}$ microobjective, linearlypolarized radiation of a helium-neon laser with the light power $P=300 \mu \mathrm{W}$ (waist diameter $d=16 \mu \mathrm{m}$ ) was incident on the face end of a LC element, with a patterned conductive chromium layer, at the incidence angle $\alpha=80^{\circ}$. The propagation of radiation within the LC layer was recorded, in response to the light scattered from inhomogeneities of LC, by the CCDcamera integrated with the $8 \mathrm{X}$ microobjective. Table 1 presents top-view images for propagation of laser radiation in a LC cell depending on the incident radiation polarization and on the voltage amplitude. Position of the interface for two director orientations is schematically given in the experimental photographs by a white dotted line.

TABLE I. LASER RADIATION PROPAGATION IN THE LC CELL WITH AN ELECTRICALLY INDUCED REFRACTIVE INTERFACE

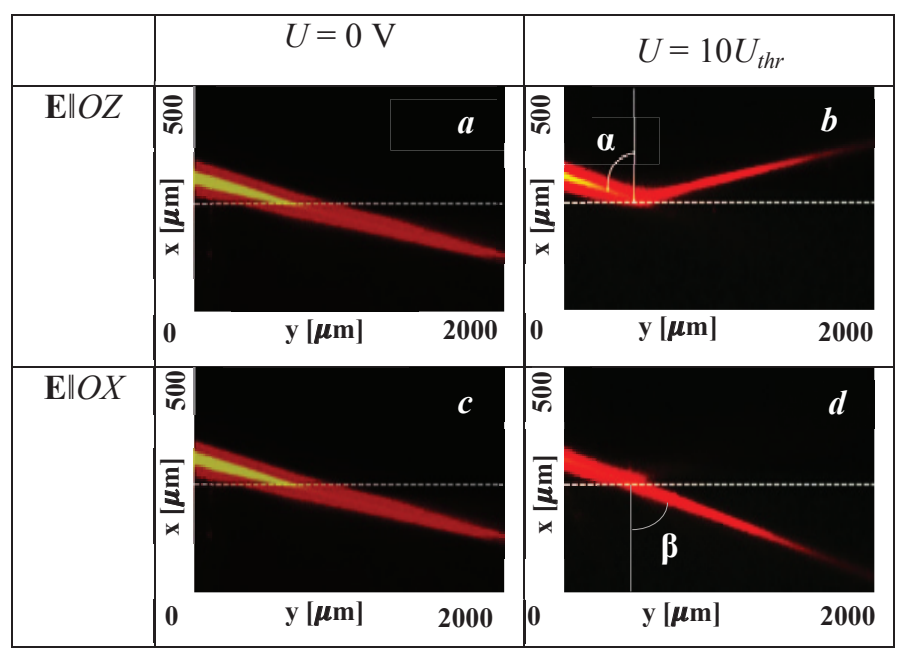

As demonstrated by the obtained experimental results, without the applied electric field, light beams are propagating rectilinear irrespective of polarization state (see Tab. 1. a,c) because molecules within the LC layer have identical planar orientation in the cell. When an external electric field is applied, within the LC layer the interface of orthogonal directions is induced for the $\mathrm{LC}$ molecules and total internal reflection of a wave with the polarization vector $\mathbf{E} \| O Z$ is realized (Tab. 1. b), whereas a wave with the polarization vector $\mathbf{E} \| O X$ passes the interface being refracted (Tab. 1. $d$ ). At the incidence angle $\alpha=80^{\circ}$ the angle of refraction comes to $\beta=79.1^{\circ}$.

To form the interface between two orientations of the director, it is sufficient to apply to the cell with a patterned electrode the voltage that is higher than the threshold $U>U_{t h}$. The increased voltage results in molecular reorientation of LC to the homeotropic position in the opaque region of the $\mathrm{LC}$ cell with a deposited chromium layer. Further growth of the voltage leads to the electromagnetic field lines which are going beyond the conductive layer to afford molecular reorientation of LC in the region adjacent to the interface, despite the absence of a conductive layer on the lower substrate of the cell.

Molecular reorientation of LC close to the interface of the opaque conductive layer of chromium was studied at the increased amplitude of an external electric field - the samples were photographed at crossed polaroids. Due to the use of an a.c. generator, rectangular signals were applied to the cell at the amplitude up to $30 \mathrm{~V}$. The samples were photographed by means of a CCD-camera integrated with the $20 \mathrm{X}$ microobjective. Fig. 2 shows experimental photographs for the displaced interface of the orthogonal director orientations, where a white dashed line indicates the edge of the etched opaque conductive layer of chromium. The photographs demonstrate that, as the voltage is growing, a bright area in the 
region without the electrode is broadened and the disclination is more displaced from the edge of chromium. Such a bright area near the electrode is formed due to molecular reorientation from planar to the homeotropic position.

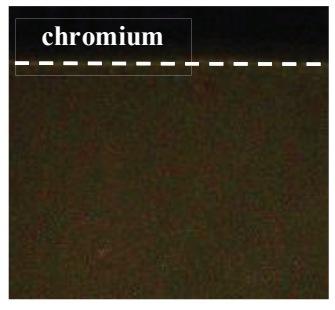

$U=0 \mathrm{~V}$

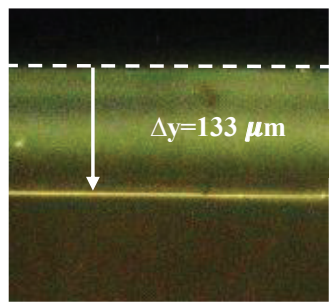

$U=20 \mathrm{~V}$

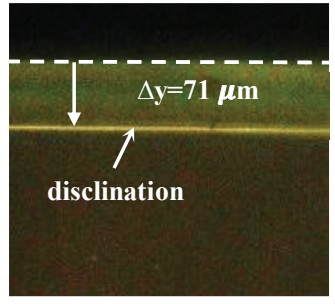

$U=10 \mathrm{~V}$

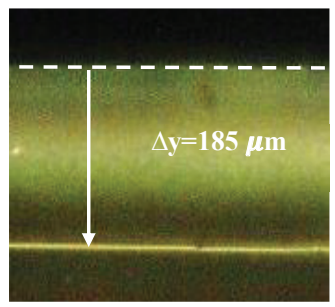

$U=30 \mathrm{~V}$
Fig. 2. Refractive interface displacement due to an external electric field

The disclination displaced to the cell region without chromium points to the fact that, with the increased voltage amplitude, more and more molecules are aligned along the field lines in accordance with the Fredericks effect [9] and are responsible for the displaced interface between homeotropic and planar orientations of the director. Spatial displacement of the disclination determines a new position of the interface between the LC director orientations.

As seen in experimental photographs in Fig. 2, an increase in the voltage amplitude leads to displacement of the director orientation interface to the cell region without the electrode. The interface displacement $\Delta y$ as a function of the voltage amplitude $U$ is shown in Fig. 3, where experimental data are given by dots, whereas the dashed line represents a linear approximation of the experimental results.

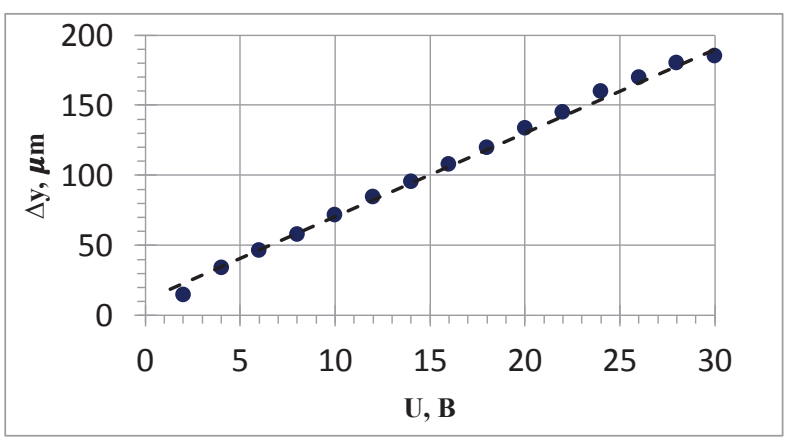

Fig. 3. Interface displacement as a function of the voltage amplitude

It is seen that, when the voltage amplitude is increased by $1 \mathrm{~V}$, the interface is displaced to a distance of $\sim 7 \mu \mathrm{m}$.
According to the experimental data in Fig. 2, the higher the amplitude of the applied voltage for the cell, the wider the bright area, where there is no reflection. Therefore, a linearly polarized light beam $(\mathbf{E} \| O Z)$ should passes the greater distance within the LC volume before it can be subjected to total internal reflection. In the process the reflection point of laser radiation is spatially displaced to the cell region, where the conductive layer is etched. Such a displacement determines variations in the reflected beam trajectory in conditions of total internal reflection that is resultant in the parallel displacement.

Fig. 4 demonstrates the propagation pattern of laser radiation for three values of the voltage amplitude obtained by overlapping the experimental photographs. Propagation of electromagnetic radiation within the LC volume was recorded at the following voltages: $U=10 \mathrm{~V}, U=20 \mathrm{~V}, U=30 \mathrm{~V}$. A white dashed horizontal line in the photo marks the edge of the opaque conductive layer of chromium. The experimental results demonstrate the displacement of the radiation of a helium-neon laser caused by the displacement of the interface between the two LC regions with the orthogonal director orientations.

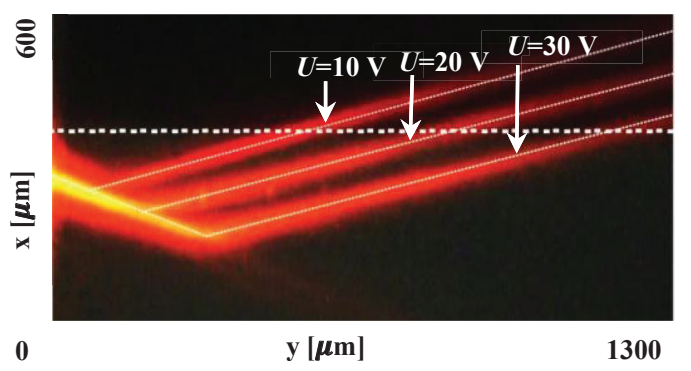

Fig. 4. - Laser beam steering by dual-domain LC cell (overlapping of 3 independent images)

It should be noted that, due to application of $30 \mathrm{~V}$ to the cell, the reflection point was displaced into the transparent region of the cell to the distance $185 \mu \mathrm{m}$, while a thickness of the LC layer was only $20 \mu \mathrm{m}$.

\section{CONCLUSION}

Thus, this paper presents the experimental studies of the propagation of linearly polarized laser radiation in the LC cell with a patterned conductive layer depending on the applied electric field. It has been found that, with a growth of the external electric field amplitude, in LC elements the refractive interface is linearly displaced to the cell region without the conductive layer. Steering of the light wave reflected from the refractive interface has been demonstrated with the proportionality factor $\sim 7 \mu \mathrm{m} / \mathrm{V}$ between the displacement and the voltage amplitude.

The revealed effect of the refractive interface displacement for orthogonal orientations of the LC director due to increase in the amplitude of an electric field applied to the LC sample offers much promise for design and manufacturing of competitive integrated-optics components aimed at microscanning. Besides, it may be used to develop modern control systems for optical radiation 


\section{ACKNOWLEDGMENT}

This work was supported by the Belarusian State Program of Scientific Investigations "Convergence-2020" ( task 3.03.5.)

\section{REFERENCES}

[1] V.G. Chigrinov, Liquid Crystal Photonics: Engineering Tools, Techniques and Tables, NY, USA: Nova Science Pub Inc., 2014.

[2] J. Beeckman, K. Neyts, P. Vanbrabant, "Liquid-crystal photonic applications", Opt. Eng. 2011. Vol. 50. No 8. P. 08120201-0820217.

[3] B. Li, S.J. Chua, Optical switches: Materials and design, India: Woodhead Publishing Limited, 2010.

[4] M. Peccianti, A. Dyadyusha, M. Kaczmarek, G. Aassanto, "Tunable refraction and reflection of self-confined light beams", Nature Physics. 2006. Vol. 2. P. 737-742.
[5] A. Komar, A. Tolstik, E. Melnikova, A. Muravsky, "Optical switch based on the electrically controlled liquid crystal interface", Appl. Opt. 2015. Vol. 54. No 16. P. 5130-5135.

[6] H.-S. Kwok, S. Naemura, H.L. Ong, Progress in Liquid Crystal Science and Technology, Singapore: World Scientific Publishing Company, 2013.

[7] V.S. Mikulich, An.A. Murawski, Al.A. Muravsky, "Influence of methyl substituents on azo-dye photoalignment in thin films" Journal of Appl. Spectr. 2016, Vol. 83. No 1. P. 115-120.

[8] I.I. Rushnova, E.A. Melnikova, A.L. Tolstik, A.A. Muravsky, "Electrically switchable photonic liquid crystal devices for routing of a polarized light wave" Opt. Commun. 2018. Vol. 413. P. 179-183.

[9] L. M. Blinov, Structure and Properties of Liquid Crystals, Springer, 2011. 